\title{
The influence of lutein supplementation on macular pigment optical density in patients with dry AMD compared with healthy control subjects
}

\author{
Semira Kaya ${ }^{1 *}$, Günther Weigert ${ }^{2}$, Nikolaus Dragostinoff ${ }^{3}$, René Werkmeister ${ }^{3}$, Berthold Pemp ${ }^{1,2}$, Stefan Sacu ${ }^{2}$, \\ Gerhard Garhöfer ${ }^{1}$, Ursula Schmidt-Erfurth², Leopold Schmetterer ${ }^{1,3}$ \\ From 16th Scientific Symposium of the Austrian Pharmacological Society (APHAR) \\ Vienna, Austria. 25-27 November 2010
}

\section{Background}

Lutein and zeaxanthin are carotenoids that are selectively taken up into the macula of the eye, where they may protect against development of age-related macular degeneration (AMD). Past studies have reported an agedependent decline of macular pigment optical density (MPOD) in patients with AMD. In the present study we investigated the age-dependence of MPOD and melatonin using spectral fundus reflectance. In addition, we hypothesized that patients with AMD have a reduced MPOD as compared to healthy controls.

\section{Methods}

A total of 82 healthy subjects and 96 patients with AMD were included in this study. The healthy control subjects showed a wide range of ages (mean 51.6 years, range 21-79 years). Patients with AMD were significantly older (mean 71.2 years, range 50-89 years). Spectral fundus reflectance of the fovea was measured in a $2.3^{\circ}$ detection field with a custom-built fundus reflectometer. Calculation of MP and melanin optical density was based on a previously published fundus reflectance model [1].

\section{Results}

No age dependence of MPOD $(r=-0.15)$ or melatonin optical density $(r=0.08)$ was found in the healthy control group. Patients with AMD, however, showed a reduced MPOD $(0.35 \pm 0.12)$ as compared to the healthy control group $(0.39 \pm 0.12, \mathrm{p}=0.013)$. No significant difference in melatonin optical density was observed between the two groups (AMD: $1.05 \pm 0.24$; healthy controls: $1.03 \pm 0.24, \mathrm{p}=0.58)$.

\section{Conclusions}

A variety of studies showing a decline in MPOD with age are based on heterochromatic flickerphotometry data. By contrast, most data indicating no change in MPOD with age are based on the use of objective technology such as fundus reflectance or autofluoresence. The present study confirms the latter studies. In addition, the data of the present study indicate that patients with AMD have reduced MPOD, but not melatonin optical density. This supports the hypothesis that reduced macular pigment is associated with AMD.

\section{Author details}

${ }^{1}$ Department of Clinical Pharmacology, Medical University of Vienna, 1090 Vienna, Austria. ${ }^{2}$ Department of Ophthalmology, Medical University of Vienna, 1090 Vienna, Austria. ${ }^{3}$ Center for Biomedical Engineering and Physics, Medical University of Vienna, 1090, Vienna, Austria.

Published: 16 November 2010

\section{Reference}

1. van de Kraats J, Berendschot TT, van Norren D: The pathways of light measured in fundus reflectometry. Vision Res 1996, 36:2229-2247.

doi:10.1186/1471-2210-10-S1-A40

Cite this article as: Kaya et al:: The influence of lutein supplementation on macular pigment optical density in patients with dry AMD compared with healthy control subjects. BMC Pharmacology 201010 (Suppl 1):A40.

\footnotetext{
* Correspondence: semira.kaya@meduniwien.ac.at

'Department of Clinical Pharmacology, Medical University of Vienna, 1090

Vienna, Austria

Full list of author information is available at the end of the article
} 